Física de la Tierra

ISSN-e: 1988-2440

http://dx.doi.org/10.5209/FITE.57600

\title{
Terremotos de la Vega Baja del Segura
}

\author{
José Manuel Martínez Solares ${ }^{1}$, Josep Batlló Ortiz ${ }^{2}$
}

Recibido: 11/06/2017 / Aceptado: 10/10/2017

Resumen. Se describen los terremotos más importantes (I > VII) ocurridos en el área próxima a la Vega Baja del Rio Segura. A partir de nuevos trabajos relativos a la sismicidad de la zona y considerando los documentos históricos ya conocidos, se realiza un análisis individual de cada sismo con objeto de asignar los parámetros de localización de forma más precisa. Asimismo, se lleva a cabo una nueva evaluación de los puntos de intensidad macrosísmica, permitiendo en algunos terremotos determinar su tamaño, bien en términos de magnitud momento o de intensidad máxima.

Palabras clave: Terremotos; Vega Baja del Segura; intensidad, magnitud momento; sismicidad.

\section{[en] Earthquakes of the Vega Baja del Segura}

\begin{abstract}
This work describes the most important earthquakes (I > VII) that took place in the area close to the Vega Baja of the Segura River. From new works related to the seismicity of the area and considering the known historical documents, an individual analysis of each earthquake in order to assign the parameters of location more accurately is carried out. A new assessment of the points of macroseismic intensity, allowing in some earthquakes to determine its size, either in terms of moment magnitude or maximum intensity, is also conducted.
\end{abstract}

Key words: Earthquakes; Vega Baja basin; intensity; moment magnitude; seismicity.

Sumario. Introducción. 1. Terremotos de 1048. 2. Terremoto de 1482. 3. Terremoto de 1673. 4. Terremoto de 1829. 5. Terremoto de 1909. 6. Terremoto de 1919. 7. Conclusiones. Referencias bibliográficas.

Cómo citar: Martínez Solares, J.M., Batllo Ortiz, J. (2017). Terremotos de la Vega Baja del Segura. Física de la Tierra, Vol. 29 (2017), 121-134.

\section{Introducción}

La Vega Baja del Rio Segura está situada al sur de la provincia de Alicante, al SE de la península Ibérica, estando ligada a la evolución reciente de la cordillera Bética y por tanto a la colisión de las placas Eurasiática y Africana en un marco compresivo de dirección general N-S. La zona está dominada por la falla del Bajo Segura (también denominada de la Vega Baja) que se encuentra localizada en el extremo NE de la zona de cizalla de la Bética Oriental, siendo una de las estructuras tectónicas de la Península con mayor actividad sísmica asociada. Se trata de una zona de falla ciega sin ruptura en superficie y su traza principal tiene varios segmentos casi para-

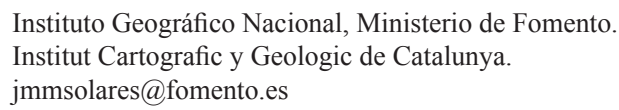


lelos entre sí de dirección ENE-WSW (Alfaro et al. 2012). Entre estos segmentos cabe destacar la falla del Bajo Segura y la falla de Torremendo, teniendo también asociadas varias fallas de dirección NW-SE (San Miguel de Salinas, Torrevieja y Guardamar). Estas fallas tienen su continuidad hacia el Este en el mar Mediterráneo. Alfaro, et al. (2012) han podido determinar tasas de desplazamiento que varían entre 0,2 y $0,4 \mathrm{~mm} / \mathrm{año.}$

La actividad sísmica de la zona es moderada, si bien terremotos de carácter destructivo se han producido con altos intervalos de recurrencia. No obstante, el reciente estudio de peligrosidad sísmica (Grupo de Trabajo IGN-UPC, 2013) ha calculado valores de la PGA similares a los obtenidos para la zona de Granada.

En el presente estudio se van a considerar algunos de los terremotos más importantes en la Vega Baja del Segura, en particular los de intensidad superior a VII (no existiendo ninguno con magnitud instrumental calculada superior a 5.0) según el catálogo sísmico del Instituto Geográfico Nacional (IGN). La localización y la intensidad de estos sismos están indicados en la tabla 1. Por su parte, la figura 1 contiene su ubicación junto con las fallas activas cartografiadas por la base de datos QAFI (IGME, 2017). Hemos de señalar que la fiabilidad de los parámetros de los sismos que se van a analizar dependen lógicamente de la información y datos disponibles, y estos son más escasos y deficientes conforme nos alejamos en el tiempo.

Tabla 1. Terremotos de la Vega Baja analizados.

\begin{tabular}{|c|c|c|c|c|}
\hline Fecha & Latitud & Longitud & Imax & Localización \\
\hline 1048 & $38^{\circ} 05^{\prime} \mathrm{N}$ & $-0^{\circ} 55^{\prime} \mathrm{W}$ & VIII & Orihuela \\
\hline 10 -oct-1482 & $38^{\circ} 05^{\prime} \mathrm{N}$ & $-0^{\circ} 55^{\prime} \mathrm{W}$ & VIII & Orihuela \\
\hline 15 -ene-1673 & $38^{\circ} 05^{\prime} \mathrm{N}$ & $-0^{\circ} 55^{\prime} \mathrm{W}$ & VIII & Orihuela \\
\hline 21 -mar-1829 & $38^{\circ} 05^{\prime} \mathrm{N}$ & $-0^{\circ} 41^{\prime} \mathrm{W}$ & IX-X & Torrevieja \\
\hline 1 -jul-1909 & $38^{\circ} 00^{\prime} \mathrm{N}$ & $-0^{\circ} 40^{\prime} \mathrm{W}$ & VII & Torrevieja \\
\hline 10 -sep-1919 & $37^{\circ} 59^{\prime} \mathrm{N}$ & $-0^{\circ} 52^{\prime} \mathrm{W}$ & VII-VIII & San Miguel de Salinas \\
\hline
\end{tabular}

Base de datos IGN

Los tres primeros terremotos de la tabla no estaban catalogados ni en Galbis (1932), ni por Mezcua y Martínez Solares (1983), ni en otros catálogos (Udías, 2015), hasta que recientes trabajos de sismicidad histórica los estudiaron, tal y como veremos más adelante. En relación con los sismos de Torrevieja de 1829 y 1909 y el de 1919 en San Miguel de Salinas, si han estado recogidos en diferentes catálogos y en algunos casos tienen estudios monográficos con más o menos amplitud. En los siguientes apartados haremos una evaluación de toda la información disponible con objeto de llegar a una mayor precisión en la localización y en la asignación del tamaño, ya sea mediante la intensidad máxima o mediante la distribución de puntos de información macrosísmica aplicando las metodologías al respecto. 


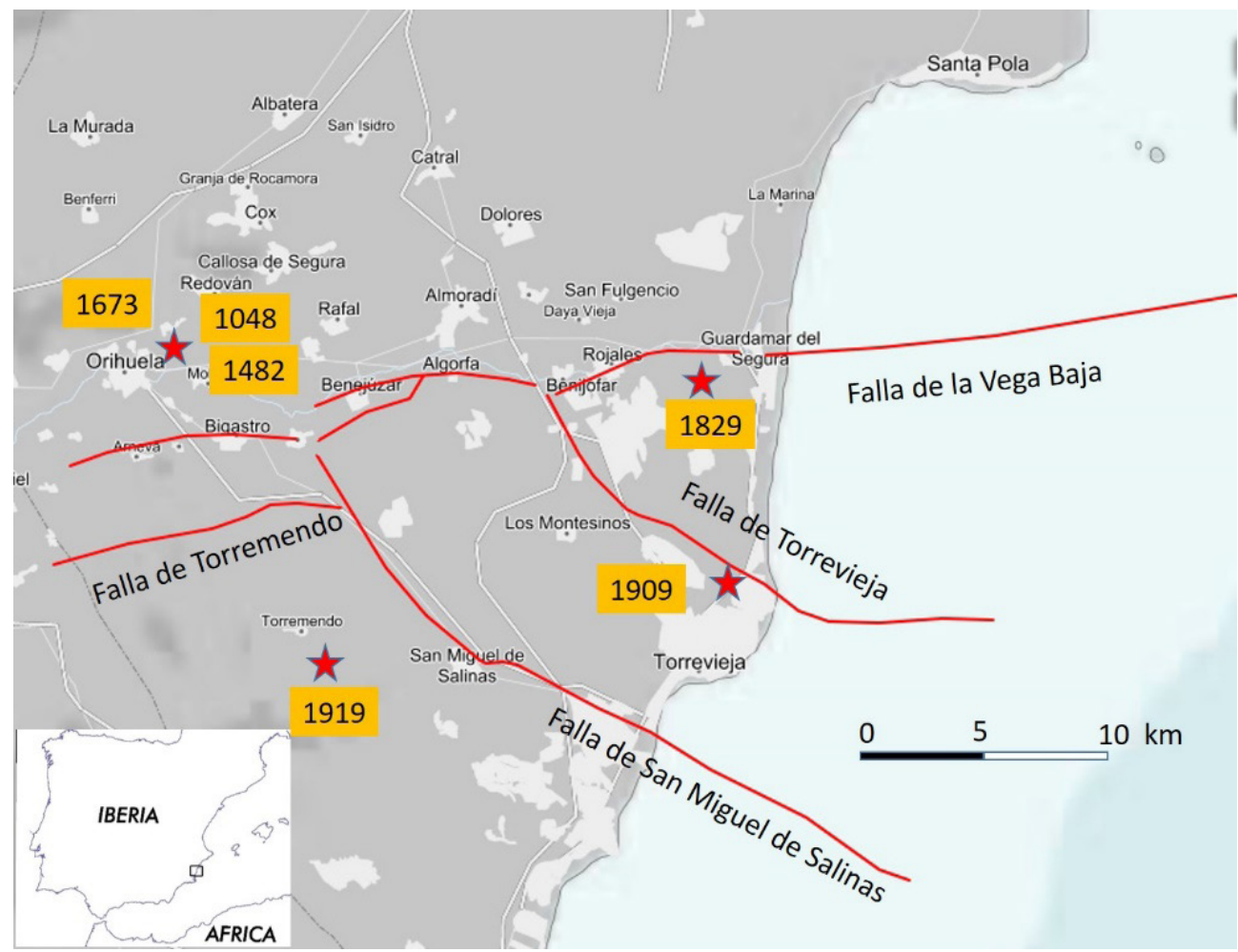

Figura 1. Terremotos de la Vega Baja del Segura de intensidad superior a VII y localización de las fallas activas presentes en la zona según QAFI (IGME, 2017).

\section{Terremoto de 1048}

Este terremoto no aparece en los catálogos clásicos (Galbis, 1932,1940; Mezcua y Martínez Solares, 1983), siendo la primera cita la incluida en el trabajo de Calvo Garcia-Tornel (1982) relativa a los movimientos sísmicos en la región murciana, siendo su fuente el geógrafo almeriense, contemporáneo con los hechos, Al-'Udri. Posteriormente otros autores han incluido este terremoto en sus trabajos sobre sismicidad histórica (Fernández Navarro-Soto, 1984; Martínez Guevara y Fernández Navarro-Soto, 1986; Vallve, 1986; Vidal, 1986; Espinar, 1994; Bretón, 1997; Martínez Solares y Mezcua, 2002).

El autor de referencia, Al-'Udri, cita los siguientes daños en las comarcas de Murcia y Orihuela:

"Las casas se derrumbaron, las torres se abatieron, así como todos los edificios altos. La mezquita mayor de Orihuela se derrumbó junto con su minarete, la tierra se abrió y muchos manantiales desaparecieron bajo tierra y surgieron otros que manaban agua hedionda." 
Posteriormente Bisbal (1984) cita un periodo sísmico entre los años 1013-1014, en el que parte del mismo documento del geógrafo Al-'Udri. Sin embargo utiliza la traducción del investigador Molina López, que equivoca la fecha del año 440 de la Hégira por la del año 404, tal y como acertadamente apuntan y corrigen Martínez Guevara y Fernández Navarro-Soto (1986), dando por tanto como valido el 1048 como año verdadero de ocurrencia.

Respecto al epicentro y a los daños, especificando genéricamente la comarca de Murcia y teniendo en cuenta la cita expresa de los efectos en Orihuela, se toma esta población como epicentro, estimándose una intensidad VIII en la escala EMS-98. Considerando que este terremoto está basado en una única fuente y siguiendo los criterios de calidad adoptados por Martínez Solares y Mezcua (2002), asignamos un factor de calidad $\mathrm{C}$ al valor de la intensidad y un valor de $\mathrm{C}$ al epicentro.

\section{Terremoto de 1482}

En principio surgen dudas respecto a la fecha de ocurrencia de un terremoto en Orihuela, bien en el año 1482 o en el 1484, dependiendo del catálogo considerado. Incluso algunos investigadores (Bisbal, 1984; Giner, 1996) recogen los dos, uno cada año. En los documentos que se disponen, el terremoto de 1482 tiene asociado el día 10 de octubre, lo que no ocurre con el de 1484 que solo figura el año.

Teniendo en cuenta los distintos catálogos y las fuentes que utiliza cada uno hemos representado en la tabla 2 un resumen de su distribución según la fecha de edición.

Tabla 2. Catálogos y fuentes de los terremotos de 1482 y/o 1484.

\begin{tabular}{|c|c|c|}
\hline Fecha sismo & Catálogo & Fuente \\
\hline 1484 & Fontseré e Iglesias, 1971 & Figueres Pacheco (1913-1925) \\
\hline 1482 & López Marinas, 1976 & Manuscrito Ayto. Orihuela (1862) \\
\hline 1484 & Rodríguez de la Torre, 1980 & Fontseré (1971), Gisbert (1903) \\
\hline 1482 & Bisbal, 1984 & $\begin{array}{c}\text { López Marinas (1976) } \\
\text { P. Bellot (1622), Gisbert (1903) }\end{array}$ \\
\hline 1484 & Fdez. Navarro-Soto, 1984 & Archivo Municipal de Orihuela (1482) \\
\hline 1482 & Giner, 1996 & Bisbal (1984) \\
\hline 1482 & Martínez Solares y Mezcua, 2002 & $\begin{array}{c}\text { Fdez. Navarro-Soto (1984), Bisbal (1984) } \\
\text { López Marinas (1976) }\end{array}$ \\
\hline 1482 &
\end{tabular}

Nota: Algunos documentos de la Fuente no han estado disponibles

El primer autor que cita los dos terremotos es Bisbal (1984) ya que sus fuentes son diferentes para cada uno de ellos. La fuente principal del sismo de 1484 es P. Bellot (1622) que en su obra Anales de Orihuela solo cita este terremoto. La cita de Bellot respecto de los daños es (ver Bisbal): "Hubo tal terremoto que todos los edificios principales se caían, y se mandó derribar las torres o reforzar las que se pudiesen, 
como la de sala, la de Bartolomé Togores, la de Juan Abret y otras." Considerando la fiabilidad que da el documento presentado por Fernández Navarro-Soto (1984) relativo al Archivo Municipal de Orihuela redactado en el mismo año del terremoto de 1482, cuyo texto, traducido al castellano, sería: "En el mes de octubre de este año y España, gran terremoto en esta ciudad que muchos edificios fueron resentidos, fue el 10 de octubre".

La información aportada por Bellot es más explícita que esta última, pero en ningún caso son contradictorias. Por tanto concluimos que se trata de un solo terremoto ocurrido el 10 de octubre de 1482 con epicentro en Orihuela por ser la única localidad con información y con intensidad VIII (EMS-98). Con los criterios de calidad anteriormente reseñados, se asigna la clase $\mathrm{C}$ tanto para el epicentro como para la intensidad.

\section{Terremoto de 1673}

Este terremoto tampoco ha estado incluido en los catálogos hasta que Bisbal (1984) lo recoge de un trabajo publicado en 1961 por el historiador Vidal Tur cuyas fuentes son el Archivo del Obispado de Orihuela-Alicante. Este autor escribe lo siguiente:

\footnotetext{
"Corrían los primeros días de Enero del año 1673 y con relativa frecuencia Orihuela y su comarca veíanse atacados por algunos movimientos sísmicos. Llegado el día 15 de dicho mes y año sobrevino uno horrible, que destruyó edificios de la Ciudad, de entre los cuales sufrió considerablemente el templo del Pilar (Santa Cruz) que quedó completamente en ruinas.

El Prelado Señor Vergé, con celo y actividad, se interesó sobremanera por la reparación de los templos afectados. El de Santa Cruz, dos años después, 1675, quedaba, aunque algo más reducido, totalmente reedificado."
}

Con la información anterior, al igual que Bisbal (1984), concluimos que se trata de un terremoto de intensidad VIII (EMS-98) y cuyo epicentro estaría situado en la única localidad citada Orihuela. Las calidades serian de tipo C para ambos parámetros.

\section{Terremoto de 1829}

El terremoto de 21 de marzo de 1829 es seguramente el mayor terremoto ocurrido en el sureste de la península Ibérica al menos en los últimos mil años. Ha sido estudiado por diversos autores desde su ocurrencia: Larramendi (1829), López Marinas (1976), Muñoz et al. (1983), Rodríguez de la Torre (1984) o Martínez Solares y Mezcua (2002). El movimiento sísmico arruinó completamente, entre otros, los pueblos de Torrevieja, Almoradí, Rojales, Guardamar del Segura y Benejuzar, ocasionando 389 víctimas mortales. Como fenómenos sismogeológicos produjo licuefacción, grietas en el terreno y se observó también alteración en el caudal de las fuentes.

El informe técnico realizado por el ingeniero Larramendi (1829) concluyó que las ciudades destruidas debían ser reconstruidas con una planta nueva, dotadas de 
regularidad y espaciosidad, con calles de un ancho comprendido entre 12 y 15 metros. Para la reconstrucción de Torrevieja, se planteó el efecto de suelo por lo que se consideró necesario trasladar la ciudad a una nueva ubicación situada a poco más de $3 \mathrm{~km}$, pero por motivos económicos decidieron reedificar la ciudad en el mismo lugar. Respecto a la construcción de las nuevas casas, el ingeniero Larramendi ya anotaba sobre el concepto de la sencillez como norma general en las construcciones sismorresistentes.

A partir de los documentos recogidos en los trabajos de Larramendi, López Marinas y Rodríguez de la Torre, en el trabajo de Martínez Solares y Mezcua (2002) se realiza una nueva evaluación macrosísmica, asignando valores de intensidad en la escala EMS-98 (Figura 3). Las intensidades máximas de grado IX-X asignadas corresponden a los pueblos de Almoradi, Benejuzar, Formentera del Segura, Torrelamata y Torrevieja. Con estos valores, más los dados para el resto de localidades por Martínez Solares y Mezcua, se han calculado el epicentro y la magnitud momento usando las metodologías de Bakun y Wentworth (1997) y de Gasperini, et al. (1999), usando diferentes modelos de atenuación. En concreto, y para el método BOXER de Gasperini se han utilizado dos modelos de atenuación diferentes que se consideran relevantes para el área de estudio. El primero es el obtenido para la península Ibérica en el proyecto NERIES-NA4 (Stucchi et al., 2010) y el segundo es el obtenido para las Béticas por Gómez-Capera et al. (2015).

Como datos macrosísmicos para el cálculo se han utilizado dos conjuntos de puntos. Uno compuesto por todas las localidades a los que fue posible asignar un valor de intensidad determinado, que sumaron en total 42 puntos. Otro, más amplio, en donde se han añadido por una parte las localidades en las que se tiene constancia de que el terremoto fue sentido (marcados con $\mathrm{S}$ en el mapa), pero que ha sido imposible asignar un valor de intensidad determinado y que hemos estimado sería aproximadamente de IV. En este caso, para incluirlos en el cálculo, se asignaron a estos puntos un valor ficticio de intensidad de 4,1. Este valor se designa para distinguirlo del 4,0 correspondiente a la intensidad IV y cuya decima no influye prácticamente en el cálculo. Por otro lado, también se añadieron algunos pueblos más en donde se sabe que se produjeron daños ( $\mathrm{D}$ en el mapa) en general pequeños, de grado 1, como caída de chimeneas o desperfectos en tabiques. A estos puntos se les asignaron un valor de 5,2 para distinguirlos de la intensidad V real. En este caso el conjunto de puntos utilizado ascendió a los 67.

Los resultados obtenidos por esta metodología, coordenadas geográficas y sus incertidumbres y la magnitud momento Mw, para los dos tipos de atenuación y las dos colecciones de puntos, se representan en la Tabla 3. Como ya es sabido a partir de las condiciones de cálculo, las coordenadas geográficas del epicentro y sus incertidumbres no varían ni con las diferentes atenuaciones ni con el número de puntos considerados, solamente la magnitud Mw varía dependiendo de estos. 


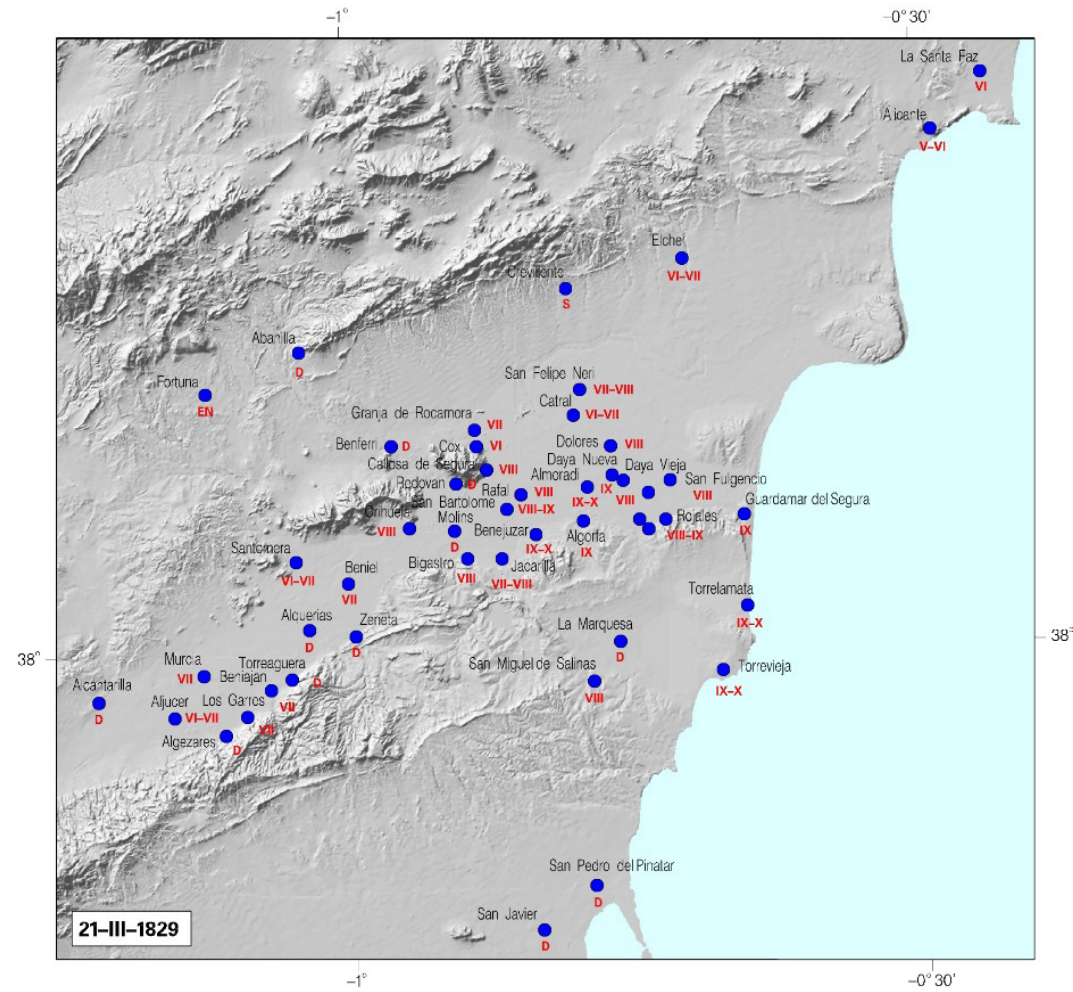

Figura 3. Localidades con información macrosísmica del terremoto de 1829. (Un listado con el resto de localidades se puede obtener de Martínez Solares y Mezcua, 2002).

Tabla 3. Resultados del programa BOXER

\begin{tabular}{|c|c|c|c|c|c|}
\hline Atenuación & $\mathbf{N}^{\mathbf{0}}$ puntos & Latitud & Longitud & Incertidumbre & Mw \\
\hline \multirow{2}{*}{ NERIES-NA4 } & 67 & 38,091 & $-0,749$ & $\Delta \mathrm{x}=5,8 \mathrm{~km} ; \Delta \mathrm{y}=2,2 \mathrm{~km}$ & $5,50 \pm 0,38$ \\
\cline { 2 - 7 } & 42 & 38,091 & $-0,749$ & $\Delta \mathrm{x}=5,8 \mathrm{~km} ; \Delta \mathrm{y}=2,2 \mathrm{~km}$ & $5,76 \pm 0,36$ \\
\hline \multirow{3}{*}{ Capera et al. } & 67 & 38,091 & $-0,749$ & $\Delta \mathrm{x}=5,8 \mathrm{~km} ; \Delta \mathrm{y}=2,2 \mathrm{~km}$ & $6,01 \pm 0,25$ \\
\cline { 2 - 7 } & 42 & 38,091 & $-0,749$ & $\Delta \mathrm{x}=5,8 \mathrm{~km} ; \Delta \mathrm{y}=2,2 \mathrm{~km}$ & $6,12 \pm 0,05$ \\
\hline
\end{tabular}

Para el método de Bakun y Wentworth (1997) se han utilizado dos modelos de atenuaciones diferentes y plenamente adecuados a la zona de interés, por una parte el de Mezcua et al. $(2004,2013)$ y por otra, el obtenido para la península Ibérica en el proyecto NERIES NA4 (Stucchi et al., 2010). El primer modelo de atenuación es lineal y el segundo incluye términos logarítmicos. Por otra parte, el método de Bakun y Wentworth es muy sensible a los puntos anómalos, es decir aquellos muy alejados del conjunto de puntos o que tienen valores de intensidad muy diferentes de 
los puntos que le rodean. Por este motivo, hemos aplicado el método a los dos conjuntos de puntos utilizados para BOXER con la salvedad que el conjunto reducido consta de 41 puntos al eliminar un punto de intensidad I (Alhama de Murcia). Los resultados obtenidos para las coordenadas del epicentro y de la magnitud momento para los dos tipos de atenuación y número de puntos están representados en la Tabla 4. Como se refleja en la tabla, tanto las coordenadas epicentrales como la Mw son significativamente muy distintas dependiendo del conjunto de puntos y modelo de atenuación considerado.

Tabla 4. Resultados del método de Bakun \& Wentworth

\begin{tabular}{|c|c|c|c|c|}
\hline Atenuación & $\mathbf{N}^{\mathbf{0}}$ puntos & Latitud & Longitud & Mw \\
\hline \multirow{2}{*}{ Mezcua et al. } & 67 & 38,08 & $-0,77$ & 5,8 \\
\cline { 2 - 5 } & 41 & 38,05 & $-0,72$ & 6,3 \\
\hline \multirow{2}{*}{ NERIES NA4 } & 67 & 38,08 & $-0,77$ & 5,4 \\
\cline { 2 - 5 } & 41 & 38,05 & $-0,72$ & 5,8 \\
\hline
\end{tabular}

Las conclusiones de este nuevo estudio son que los resultados obtenidos con el conjunto de puntos reducido son más estables que los obtenidos con más puntos inferidos a partir de hipótesis de trabajo. Se hace evidente que las intensidades asignadas a los casos "S" y "D" no se ajustan a nuestras suposiciones. Asimismo, comparando el grado de destrucción e intensidades asignadas a este terremoto con eventos recientes parece plausible que los resultados obtenidos con las atenuaciones propuestas por Gómez-Capera et al. (2015) y Mezcua et al. (2004) se ajustan mejor a la realidad. Por tanto la magnitud momento equivalente del terremoto de Torrevieja será ligeramente mayor que 6, pudiéndola situar en el intervalo 6.1-6.3.

\section{Terremoto de 1909}

En el mes de febrero de 1909 se registra una primera actividad sísmica en la provincia de Alicante cuya zona epicentral estaría próxima a Crevillente y percibido con intensidad VI (Andreu, 1910), características que no obstante le excluyen del presente trabajo.

Es el día 30 de junio de 1909 cuando se inicia una segunda actividad de mayor intensidad, ya próxima a Torrevieja, con un sismo premonitorio de grado III y seguido de un terremoto principal el día siguiente, 1 de julio, con intensidad VII y que analizaremos a continuación. La tabla 5 refleja los terremotos de este periodo obtenidos del catálogo de Mezcua \& Martínez Solares (1983) cuyas fuentes principales son Sánchez Navarro-Neumann (1921) y Galbis (1932), que a su vez recogen la información de Jiménez de Cisneros (1909) y Andreu (1910). Asimismo, con los datos aportados en estos trabajos para el terremoto principal se publicó un mapa de isosistas (Mezcua, 1982). 
Tabla 5. Serie sísmica de 1909 en Torrevieja (Mezcua y Martinez Solares, 1983)

\begin{tabular}{|c|c|c|c|c|c|}
\hline Fecha & Hora & Intensidad & Fecha & Hora & Intensidad \\
\hline 30-junio & $18: 30$ & III & 19-julio & $22: 45$ & III \\
\hline 1-julio & $14.12: 18$ & VII & 20-julio & $21: 30$ & III \\
\hline 2-julio & $00: 52$ & V & 25-julio & $04: 45$ & III \\
\hline 2-julio & $01: 45$ & V & 30-julio & $10: 35$ & IV \\
\hline 2-julio & $03: 00$ & IV & 4-agosto & $12: 30$ & IV \\
\hline 2-julio & $10: 00$ & V & 9-agosto & - & V \\
\hline 2-julio & $12: 02: 20$ & VI & 10-agosto & - & V \\
\hline 2-julio & $23: 00$ & IV & 14-agosto & - & III \\
\hline 3-julio & $05: 30$ & III & 16-agosto & - & III \\
\hline 4-julio & $16: 30$ & III & 28-agosto & - & V \\
\hline 5-julio & $10: 00$ & IV & & & \\
\hline
\end{tabular}

Los datos de información macrosísmica dados por Galbis están referidos a la escala Forel-Mercalli. La equivalencia de esta escala con la EMS-98 (Grunthal, 1998) la podemos obtener primero de Munuera (1963) para el paso a la de Mecalli y posteriormente de esta a la EMS-98 mediante Musson et al. (2010). Hasta el grado VII las tres escalas mencionadas son equivalentes, lo que nos permite asumir los valores dados por Galbis como pertenecientes a la escala EMS-98 y que serían los reflejados en la tabla 5.

Llama la atención la diferencia en la asignación de intensidades del terremoto principal entre los diferentes autores. En la tabla 6 figuran los grados por localidades.

Tabla 6. Asignación de intensidades por autores del terremoto de 1 de julio.

\begin{tabular}{|c|c|c|c|}
\hline Localidad & Intensidad & Autor & Este trabajo \\
\hline Torrevieja & $\begin{array}{c}\text { VII } \\
\text { VI-VII }\end{array}$ & $\begin{array}{c}\text { Jiménez de C., Galbis } \\
\text { Sánchez N-N, Andreu }\end{array}$ & VI \\
\hline Torrelamata & $\begin{array}{c}\text { VII } \\
\text { VI-VII }\end{array}$ & $\begin{array}{c}\text { Jiménez de C., Galbis } \\
\text { Sánchez N-N, Andreu }\end{array}$ & VI \\
\hline Guardamar del Segura & $\begin{array}{c}\text { VII } \\
\text { VI-VII }\end{array}$ & $\begin{array}{c}\text { Jiménez de C., Galbis } \\
\text { Sánchez N-N, Andreu }\end{array}$ & VI \\
\hline Rojales & VI & Los cuatro autores & V \\
\hline Benijofar & VI & Los cuatro autores & V \\
\hline Pilar de la Horadada & VI & Sánchez N-N, Andreu & V \\
\hline Benejuzar & VI & $\begin{array}{c}\text { Jiménez de C., Galbis } \\
\text { Andreu }\end{array}$ & V \\
\hline Jacarilla & $\begin{array}{c}\text { VI } \\
\text { V-VI }\end{array}$ & $\begin{array}{c}\text { Jiménez de C., Galbis } \\
\text { Andreu }\end{array}$ \\
\hline
\end{tabular}




\begin{tabular}{|c|c|c|c|}
\hline Localidad & Intensidad & Autor & Este trabajo \\
\hline Almoradí & $\begin{array}{c}\text { V-VI } \\
\text { V }\end{array}$ & $\begin{array}{c}\text { Andreu } \\
\text { Jiménez de C., Galbis }\end{array}$ & V \\
\hline Dolores & $\begin{array}{c}\text { V-VI } \\
\text { V }\end{array}$ & $\begin{array}{c}\text { Andreu } \\
\text { Jiménez de C., Galbis }\end{array}$ & V \\
\hline San Fulgencio & $\begin{array}{c}\text { V-VI } \\
\text { V }\end{array}$ & $\begin{array}{c}\text { Andreu } \\
\text { Jiménez de C., Galbis }\end{array}$ & $\mathrm{V}$ \\
\hline Daya Nueva & $\mathrm{V}$ & Jiménez de C., Galbis & IV \\
\hline Daya Vieja & $\mathrm{V}$ & Jiménez de C., Galbis & IV \\
\hline Bigastro & $\mathrm{V}$ & Jiménez de C., Galbis & IV \\
\hline Orihuela & IV & $\begin{array}{c}\text { Jiménez de C., Andreu, } \\
\text { Galbis }\end{array}$ & III \\
\hline Benferri & IV & Jiménez de C., Andreu & III \\
\hline Callosa de Segura & IV & Andreu & III \\
\hline Crevillente & IV & $\begin{array}{c}\text { Jiménez de C., Andreu, } \\
\text { Galbis }\end{array}$ & III \\
\hline Elche & IV & $\begin{array}{c}\text { Jiménez de C., Andreu, } \\
\text { Galbis }\end{array}$ & III \\
\hline Santa Pola & IV & Andreu & III \\
\hline Alicante & IV & Andreu, Galbis & II-III \\
\hline S. Pedro del Pinatar & Fuerte & Andreu & III-IV \\
\hline Murcia & Débil & $\begin{array}{c}\text { Jiménez de C., Andreu, } \\
\text { Galbis }\end{array}$ & II-III \\
\hline
\end{tabular}

Andreu (1910) y Sánchez Navarro-Neumann (1921) dan una intensidad máxima de VII, mientras que Jiménez de Cisneros (1909) y Galbis (1932) asignan VI-VII. La información disponible sobre los efectos causados, aunque limitada, nos puede permitir precisar el valor de la intensidad. Sánchez Navarro-Neumann los describe así en Torrevieja: "El más fuerte fue el de las 14 del 1 de julio, que derribo un tejado y uno de los muros de una casa bastante buena y causó averías en otras, con intenso pánico". El diario $\mathrm{ABC}$ de fecha 2 de julio en su página 10 anota lo siguiente: "En Torrevieja fue más intenso, agrietándose algunos edificios."

Por su parte, Andreu aporta la siguiente información. "En Torrevieja, Torre la Mata y Guardamar, los efectos producidos consistieron en fuertes crujidos del maderamen, algunas ligeras grietas en los edificios y desprendimientos en los cielos rasos y revestimiento de los muros, no contándose otros de mayor importancia a pesar de la violencia de la sacudida". En otro apartado de su trabajo describe el siguiente fenómeno sismogeológico: "Como efectos producidos por el sismo, se formó en la costa frente a Guardamar unas depresiones, de cuatro a cinco metros de diámetro y entre uno y dos de profundidad. Estos hundimientos se han presentado siempre que en la zona se ha experimentado un terremoto de consideración y en el mismo sitio y dirección; esto es, a lo largo de la costa arenosa, en una longitud de dos kilómetros y 
a una distancia variable entre quince y veinticinco metros de la línea de costa batida por las aguas, contándose hasta diez hundimientos en algunos casos".

Teniendo en cuenta los datos anteriormente señalados y a partir de la escala EMS98 (Grunthal, 1998) la intensidad del sismo principal en la zona epicentral debería ser de VI, inferior a las asignadas en los trabajos contemporáneos al terremoto. La experiencia en el análisis de este tipo de información macrosísmica nos dice que antes de existir las escalas que diferenciaban claramente los tipos de vulnerabilidad, de grado de daño o de estimaciones de cantidad, los sismólogos asignaban el valor puntual máximo, lo cual evidentemente sobreestimaba la intensidad macrosísmica. En consecuencia rebajamos también los valores de intensidad asignados al resto de localidades de la tabla 4 (columna derecha), pues además la mayoría de ellos estaban estimados a partir del ruido.

Resumiendo, para el terremoto principal del 1 de julio a las 14:12 modificamos el epicentro respecto al catálogo del IGN, ubicándolo al norte de Torrevieja, en las coordenadas $38^{\circ} 03^{\prime} \mathrm{N}$; 0 $0^{\circ} 39^{\prime} \mathrm{W}$, e intensidad máxima de VI (EMS-98).

\section{Terremoto de 1919}

El 10 de septiembre de 1919 comenzó en el área de Jacarilla y Torremendo una serie sísmica con la ocurrencia de dos terremotos principales que sucedieron en un corto intervalo de 16 minutos (10:40 y las 10:56 GMT) y que durante las semanas siguientes fueron seguidos de numerosas réplicas. Sánchez Navarro-Neumann (1919a, 1919b), Kindelán y Gorostizaga (1920), Inglada (1926, 1927), Rey Pastor (1943) publicaron estudios instrumentales y macrosísmicos de estos terremotos. Recientemente Garrido (2012) recopila la información disponible y la amplia con nuevos documentos. En la tabla 7 se muestran sus resultados para los dos terremotos principales, los cuales son los que actualmente tiene recogidos la base de datos del IGN.

Tabla 7. Sismos principales del 10 de septiembre de 1919.

\begin{tabular}{|c|c|c|c|c|c|}
\hline Fecha & Hora & Latitud & Longitud & Io & Magnitud \\
\hline 10-sep-1919 & $10: 40: 31$ & $37,9833 \mathrm{~N}$ & $0,8667 \mathrm{~W}$ & VII-VIII & 5,2 \\
\hline 10-sep-1919 & $10: 56: 44$ & $37,9833 \mathrm{~N}$ & $0,8667 \mathrm{~W}$ & VII & 5,1 \\
\hline
\end{tabular}

Base de datos IGN

Posteriormente Batllo, et al. (2015) realizan un nuevo estudio a partir de los registros instrumentales en 11 estaciones sísmicas y con nueva información macrosísmica contenida en una copia de un informe manuscrito que en el momento del terremoto fue llevado a cabo por ingenieros del IGN. El estudio evalúa de nuevo los datos macrosísmicos en la escala EMS-98 y a partir de los puntos de intensidad determina el epicentro y la magnitud momento usando las metodologías de Bakun y Wentworth (1997) y de Gasperini et al. (1999), usando diferentes modelos de atenuación. Asimismo, con los registros de las estaciones determina por un lado el epicentro instrumental y por otro las magnitudes momento de los dos terremotos principales del día 10. Analizando los resultados y valorando las diferentes soluciones que se plantean, 
tomamos como mejores opciones para los dos sismos los parámetros que se reseñan en la tabla 8 .

Tabla 8. Soluciones adoptadas para los sismos del 10 de septiembre de 1919.

\begin{tabular}{|c|c|c|c|c|c|}
\hline Fecha & Hora & Latitud & Longitud & Imax & Magnitud \\
\hline 10-sep-1919 & $10: 40: 31$ & $38,06 \mathrm{~N}$ & $0,80 \mathrm{~W}$ & VII-VIII & 5,5 \\
\hline 10-sep-1919 & $10: 56: 44$ & $38,06 \mathrm{~N}$ & $0,80 \mathrm{~W}$ & VII & 5,3 \\
\hline
\end{tabular}

\section{Conclusiones}

La revisión realizada ha permitido modificar algunos parámetros de localización y/o tamaño respecto a los catálogos anteriores, sobre todo en los sismos recientes, donde la información documental ha sido más numerosa y de mayor calidad. Asimismo, la disponibilidad de registros instrumentales, caso del sismo de 1919, permiten un análisis cuantitativo usando metodologías más precisas y que deberán seguir utilizándose en terremotos de similares características.

En relación con los terremotos más antiguos, los estudios presentan una gran incertidumbre en su interpretación al no disponer de suficiente información, por lo que estimamos muy necesario seguir investigando en los diferentes archivos y bibliotecas por especialistas en las diferentes épocas históricas.

\section{Referencias bibliográficas}

Alfaro, P., Bartolomé, R., Borque, M.J., Estévez, A., García-Mayordomo, J., García-Tortosa, F.J., Gil, A.J., Gràcia, E., Lo Iacono, C., Perea, H. (2012) The Bajo Segura Fault Zone: Active blind thrusting in the Eastern Betic Cordillera (SE Spain). Journal of Iberian Geology 38 (1): 271-284.

Andreu, J. (1910). Sobre los macrosismos de 1909 en la provincia de Alicante. Bol. Soc. Aragonesa de Ciencias Naturales, Febrero, p. 45-54.

Bakun, W.H. and C. M. Wentworth (1997). Estimating earthquake location and magnitude from seismic intensity data, Bull. Seism. Soc. Am., 87, 1502- 1521.

Batlló, J., Martínez-Solares, J.M., Macià, R., Stich, D., Morales, J., Garrido, L. (2015).- The autumn 1919 Torremendo (Jacarilla) earthquake series (SE Spain). Annals of Geophysics, 58, 3,S0324; doi:10.4401/ag-6686.

Bisbal Cervelló, L. (1984). Estudio de la distribución de intensidades sísmicas en el ámbito valenciano. Su incidencia en las obras públicas. Tesis Doctoral. Univ. Politécnica de Valencia. 9 Tomos.

Bretón González, M. (1997). Los terremotos en la Edad Media y sus efectos en el patrimonio histórico (siglos IX-XVI). Precisiones sobre sismicidad histórica. Tesis Doctoral. Universidad de Granada. 2 Tomos.

Calvo Garcíatornel, F. (1982). El riesgo, un intento de valoración geográfica. Acad. Alfonso X el Sabio. Murcia, p. 2535.

Espinar Moreno, M. (1994). Los estudios de sismicidad histórica en Andalucía: Los terremotos históricos de la provincia de Almería. En El estudio de los terremotos en Almería. Inst. de Estudios Almerienses, p.115180. 
Fernández Navarrosoto, S. (1984). Temblores de tierra en los núcleos sísmicos de Murcia La Alberca y Orihuela Benejuzar. Estudio de sismicidad histórica. Inst. Geográfico Nacional, Informe interno. pp. 57.

Fontsere, E. e Iglesies, J. (1971). Recopilacio de dades sismiques de les terres catalanes entre 1100 i 1906. Fundació Salvador Vives, pp. 546.

Galbis Rodríguez, J. (1932). Catálogo sísmico de la zona comprendida entre los meridianos $5^{\circ}$ E y $20^{\circ} \mathrm{W}$ y paralelos $45^{\circ}$ y $25^{\circ} \mathrm{N}$. Instituto Geográfico y Catastral, Tomo I, $807 \mathrm{pp}$.

Galbis Rodríguez, J. (1940). Catálogo sísmico de la zona comprendida entre los meridianos $5^{\circ}$ E y $20^{\circ} \mathrm{W}$ y paralelos $45^{\circ}$ y $25^{\circ} \mathrm{N}$. Instituto Geográfico y Catastral, Tomo II, $278 \mathrm{pp}$.

Garrido, L. (2012). Terremoto de Jacarilla de 10 de Septiembre de 1919 y su serie de réplicas. Trabajo Académico Dirigido, Universidad Complutense de Madrid, 30 p.

Gasperini, P., F. Bernardini, G. Valensise and E. Boschi (1999). Defining seismogenic sources from historical earthquake felt reports. Bull. Seismol. Soc. Am., 89, 94-110.

Giner Caturla, J.J. (1996).- Sismicidad y Peligrosidad Sísmica en la Comunidad Autónoma Valenciana. Análisis de incertidumbres. Tesis Doctoral, Universidad de Granada. 3 tomos.

Gomez-Capera, A.A., Rovida, A., Gasperini, P., Stucchi, M., Viganò, D. (2015). The determination of earthquake location and magnitude from macroseismic data in Europe, Bull. Earthquake Eng., 13(5): 1249-1280. doi:10.1007/s10518-014-9672-3

Grunthal, G. (ed.) (1998). European Macroseismic Scale 1998. Cahiers du Centre Europeen de Geodynamique et de Seismologie. Vol. 15, 99 pp.

Grupo de Trabajo IGN-UPM (2013). Actualización de Mapas de Peligrosidad Sísmica de España, 2012. Instituto Geográfico Nacional, 267 pags.

Instituto Geologico y Minero de España (2017). QAFI: Quaternary Active Faults Database of Iberia. V.3 Version online: http://www.igme.es/QAFI/.

Instituto Geografico Nacional (2017). Catálogo de terremotos. http://www.ign.es/web/ign/ portal/sis-catalogo-terremotos. (Último acceso mayo 2017)

Inglada, V. (1926). El sismo del bajo Segura de 10 de septiembre de 1919. Cálculo de las coordenadas del foco basado en la hora inicial de los sismogramas registrados en varias estaciones próximas, Bol. Inst. Geol. Minero, XLVIII, 35-48.

Inglada, V. (1927). Estudio de sismos españoles. El terremoto del bajo Segura de 10 de septiembre de 1919. Cálculo de su profundidad hipocentral y de la hora inicial de sus sacudidas en el foco y en el epicentro. Imprenta Clásica Española, Madrid, pp.72.

Jimenez de Cisneros, D. (1909). Notas sobre los terremotos de Alicante. Bol. R. Soc. Esp. Hist. Natural, T.IX, p. 312, 313, 372-374.

Kindelán, V. y J. Gorostizaga (1920). Sobre los terremotos ocurridos en las provincias de Alicante y Murcia en 1919. Boletín del Instituto Geológico de España. XLII, 247-277.

Larramendi, J.A. (1829). Memoria y relación circustanciada de los estragos que la terrible catástrofe de los terremotos de 21 de marzo y siguientes, principalmente, el del Sábado Santo 18 de abril hasta el presente dia, han causado en Torrevieja y demás pueblos de la gobernación de Orihuela y sus inmediaciones, en la ciudad de Murcia y algunos pueblos de la provincia de este nombre. Madrid, Imprenta Real, pp. 24.

López Marinas, J.M. (1976). Sismo de 21 de marzo de 1829. Hidroeléctrica Española, Informe interno. pp. 201.

Martínez Guevara, J.B. y Fernández Navarrosoto, S. (1986). Catálogo sísmico de la región de Murcia. (Sismicidad histórica hasta el siglo XVIII). Informe interno. Inst. Geográfico Nacional, pp. 71.

Martínez Solares, J.M. y Mezcua, J. (2002). Catalogo sísmico de la Península Ibérica (880 a.C. - 1900). Instituto Geográfico Nacional, 253 pp + 1 mapa. 
Mezcua, J. (1982). Catálogo general de isosistas de la península Ibérica. Inst. Geográfico Nacional, Pub. 202, pp. 322.

Mezcua, J. y Martínez Solares, J.M. (1983). Sismicidad del área Iberomogrebi. Instituto Geográfico Nacional, 301 pp + 1 mapa.

Mezcua, J., Rueda, J. and García Blanco, R.M. (2004). Revaluation of historic earthquakes in Spain, Seismol. Res. Lett., 75, 75-81.

Mezcua, J., Rueda, J. and García Blanco, R.M. (2013). Observed and Calculated Intensities as a Test of a Probabilistic Seismic-Hazard Analysis of Spain. Seismol. Res. Lett., 84, 772780.

Ministerio de Obras Públicas y Urbanismo (1986). Estudio de determinación de los datos sísmicos de base para las obras hidráulicas. Dir. Gral. Obras Hidráulicas, Tomo II, Anejo 4, pp. 401.

Munuera, J.M. (1963). A study of seismicity on the peninsula Iberian area. Technical Note n.1 Seismic Data. Inst. Geográfico y Catastral, pp.93.

Muñoz, D., Udias, A. y Moreno, E. (1983).- Reevaluación de los datos del terremoto de 1829 (Torrevieja). En Sismicidad histórica de la región de la península Ibérica. Asoc. Española de Ing. Sísmica, Madrid, 38-41.

Musson, R.M.W., Grünthal, G. \& Stucchi, M. (2010). - The comparison of macroseismic intensity scales. J. Seismol. 14:413-428.

Rey Pastor, A. (1943). La comarca sísmica del Bajo Segura. Servicio de Sismología. Instituto Geográfico y Catastral, Madrid.

Rodríguez de la Torre, F. (1984). Los terremotos alicantinos de 1829. Instituto de Estudios Alicantinos, Serie I, N.100, pp. 322.

Sánchez Navarro-Neumann, M. (1919a). Los temblores alicantinos de septiembre de 1919. Rev. Ibérica, XII, n. 301, 284.

Sánchez Navarro-Neumann, M. (1919b). Los terremotos sentidos en las provincias de Alicante y Murcia en septiembre de 1919. Rev. Soc. Astr. de España y América, n. 69, 85-88.

Sánchez Navarro Neumann, M. (1921). Lista de los terremotos más notables sentidos en la península Ibérica desde los tiempos más remotos, hasta 1917, inclusive, con ensayo de agrupación en regiones y períodos sísmicos. La Estación Sismológica y el Observatorio Astronómico y Meteorológico de Cartuja (Granada). Memorias y trabajos de vulgarización científica. p.1165.

Stucchi, M., Rovida, A., Gómez Capera, A.A., Musson, R., Papaioannou, CH., Batlló, J. (2010). NA4 European Earthquake Catalogue 1000-1963, $M>5.8$, Deliverable D10. NERIES, Distributed Archive of Historical Earthquake Data (NA4), EC Project number 026130, internal report, 30 pp. http://emidius.mi.ingv.it/neries_NA4/deliverables.php.

Udias, A. (2015).- Historical Earthquakes (before 1755) of the Iberian Peninsula in Early Catalogues. Seism.Res.Lett. v. 86, n. 3, May.

Vallve, J. (1986). La división territorial de la España musulmana. Cons. Sup. Inves. Científicas, Departamento de Estudios Arabes, p.123125.

Vidal Sánchez, F. (1986). Sismotectónica de la región BéticasMar de Alborán. Tesis Doctoral, Univ. Granada, pp.457. 